\title{
On Poincaré gauge theory of gravity, its equations of motion, and Gravity Probe B
}

\author{
Friedrich W. Hehl ${ }^{\mathrm{a}}$ \\ anstitute for Theoretical Physics, University of Cologne, 50923 Köln, Germany \\ and \\ Dept. of Physics and Astronomy, University of Missouri, Columbia, MO 65211, USA \\ Yuri N. Obukhov ${ }^{\mathrm{b}}$ \\ b Theoretical Physics Laboratory, Nuclear Safety Institute, Russian Academy of Sciences, B. Tulskaya 52, 115191 Moscow, Russia \\ Dirk Puetzfeld ${ }^{\mathrm{c}}$ \\ c ZARM, University of Bremen, Am Fallturm, 28359 Bremen, Germany
}

Abstract

We discuss the structure of the Poincaré gauge theory of gravity (PG) that can be considered as the standard theory of gravity with torsion. We reconfirm that torsion, in the context of PG, couples only to the elementary particle spin and under no circumstances to the orbital angular momentum of test particles. We conclude that, unfortunately, the investigations of Mao et al. (2007) and March et al. (2011) — who claimed a coupling of torsion to orbital angular momentum, in particular in the context of the Gravity Probe B (GPB) experiment - do not yield any information on torsion. File GPBtorsionPLA10.tex, 15 May 2013.

Key words: Torsion, Gravity Probe B, Poincaré gauge theory, Spin angular momentum, Equations of motion

\section{Introduction}

Ever since E.Cartan in the 1920s enriched the geometric framework of general relativity (GR) by introducing a torsion of spacetime, the question arose whether one could find a measurement technique for detecting the presence of a torsion field. Mao et al. [1] claimed that the rotating quartz balls in the gyroscopes of the GPB experiment [2], falling freely on an orbit around the Earth, should "feel" the torsion. We emphasize that the GPB team of Everitt et al. never made such a claim; they were aware that GPB would not be able to sense torsion [3]. However, similar to Mao et al., March et al. [4] argue with the precession of the Moon and the Mercury and extend later their considerations to the Lageos satellite.

Email addresses: hehl@thp.uni-koeln.de (Friedrich W. Hehl), yo@thp.uni-koeln.de (Yuri N. Obukhov),

dirk.puetzfeld@zarm.uni-bremen.de (Dirk Puetzfeld).

URLs: http://www.thp.uni-koeln.de/gravitation/ (Friedrich W. Hehl), http://puetzfeld.org (Dirk Puetzfeld).
A consistent theory of gravity with torsion emerged during the early 1960s as gauge theory of the Poincaré group, see the review in [5]. This Poincaré gauge theory of gravity incorporates as simplest viable cases the Einstein-Cartan(Sciama-Kibble) theory (EC), the teleparallel equivalent $\mathrm{GR}_{\|}$of $\mathrm{GR}$, and GR itself. So far, PG and, in particular, the existence of torsion have not been experimentally confirmed. However, PG is to be considered as the standard theory of gravity with torsion because of its very convincing gauge structure.

Since the early 1970s up to today, different groups have shown more or less independently that torsion couples only to the elementary particle spin and under no circumstances to the orbital angular momentum of test particles. This is established knowledge and we reconfirm this conclusion by discussing the energy-momentum law of PG, which has same form for all versions of PG. Therefore, we conclude that, unfortunately, the investigations of Mao et al. and March et al. do not yield any information on torsion. 


\section{Torsion defined, spin of matter introduced}

Einstein's theory of gravitation, GR, was finally formulated in 1916. Already since this time, mathematicians and physicists, namely Hessenberg, Levi-Civita, Weyl, Schouten, and Eddington, amongst others, started to develop the geometrical concept of a (linear) connection $\Gamma$. This is a tool for the parallel displacement of vectors in a differential manifold, in particular in 4-dimensional spacetime. The final formulation of the connection was given by E.Cartan in 1923/24. He defined the connection 1-form $\Gamma_{\alpha}{ }^{\beta}=\Gamma_{i \alpha}{ }^{\beta} d x^{i}$ as a new fundamental geometrical object (with $\alpha, \beta, .$. as frame and $i, j, \ldots$ as coordinate indices, both running from 0 to 3 ); for the explicit references and for the formalism, including the conventions, compare [5], pp.17-21.

If the connection is expressed purely in coordinate components, then the antisymmetric part of it is a tensor, Cartan's torsion tensor,

$$
T_{i j}{ }^{k}=\Gamma_{i j}{ }^{k}-\Gamma_{j i}{ }^{k} \equiv 2 \Gamma_{[i j]}^{k}=-T_{j i}{ }^{k},
$$

with its 24 independent components. This is the tensor alluded to in the title of our paper. Mao et al. [1] wanted to sense torsion by using the results of the Gravity Probe B experiment of Everitt et al. [2]; later, March et al. [4] tried to do the same thing by using data of the Moon, of the Mercury, and of the Lageos satellite. We will come back to this issue later.

In GR, the Riemannian connection is represented by the Christoffel symbols $\widetilde{\Gamma}_{i j}{ }^{k}:=\frac{1}{2} g^{k l}\left(\partial_{i} g_{j l}+\partial_{j} g_{l i}-\partial_{l} g_{i j}\right)$, where $g_{i j}$ are the components of the metric tensor and $\partial_{i}:=\partial / \partial x^{i}$. The Riemannian connection is symmetric, it is torsion-free, that is, $\widetilde{T}_{i j}{ }^{k}=0$. Massive test particles in GR move along the geodesics of the Riemannian connection:

$$
\frac{d^{2} x^{k}}{d \tau^{2}}+\widetilde{\Gamma}_{i j}{ }^{k} \frac{d x^{i}}{d \tau} \frac{d x^{j}}{d \tau}=0 .
$$

When Cartan extended the geometrical framework of GR by introducing a torsion of spacetime, he was conscious of the fact that he also had to use a more fine-grained description of matter than in GR. Instead of a classical fluid, acting via a symmetric energy-momentum density $\mathfrak{t}$, he suggested a Cosserat type fluid with an asymmetric energymomentum density $\mathfrak{T}$ and an intrinsic or spin angular momentum density $\mathfrak{S}$, see [5], pages 21 and 103 .

This conception has been developed even before the spin of the electron was discovered. We recognize that the introduction of the geometrical concept of a torsion goes hand in hand with ascribing to matter, besides an energymomentum density, a further dynamical characteristics, namely a spin angular momentum density. In a generalrelativistic theory of gravity, torsion and spin are interdependent.

This interdependence was clear to Cartan. However, because of an unfounded assumption, see Sec. 7, he was not able to formulate a consistent theory of gravity with torsion.

\section{Poincaré gauge theory as standard torsion theory}

In the early $1960 \mathrm{~s}$, a consistent framework for a valid physical theory of torsion was initiated by Sciama [6] and Kibble [7]. It was conceived as a gauge theory of the Poincaré group [7], the semi-direct product of the translations (4 parameters) and the Lorentz rotations (6 parameters). In Minkowski spacetime, the Poincaré group acts rigidly ("globally"). By means of the gauge procedure à la Weyl-Yang-Mills, the Poincaré group is "localized", acts merely locally. This is made possible by introducing 4 gauge potentials for the translations and 6 gauge potentials for the Lorentz rotations. The emerging theory is called Poincaré gauge theory of gravitation (PG), see [5], Part B for details.

The arena of the PG is a Riemann-Cartan (RC) spacetime. It is determined by a metric $g_{\alpha \beta}$ (and its reciprocal $g^{\gamma \delta}$ ), an orthonormal coframe $\vartheta^{\alpha}=e_{i}{ }^{\alpha} d x^{i}$, and a Lorentz connection $\Gamma^{\alpha \beta}:=g^{\alpha \gamma} \Gamma_{\gamma}{ }^{\beta}=-\Gamma^{\beta \alpha}=\Gamma_{i}^{\alpha \beta} d x^{i}$. Having such a connection, we can define a covariant exterior derivative $D$. For a RC-space, we find $D g_{\alpha \beta}=0$ (vanishing nonmetricity).

The coframe $\vartheta^{\alpha}$ can be understood as translational gauge potential and the Lorentz connection $\Gamma^{\alpha \beta}=-\Gamma^{\beta \alpha}$ as rotational gauge potential. The corresponding gravitational field strengths are torsion and curvature, respectively, which we find by differentiation of the corresponding potentials:

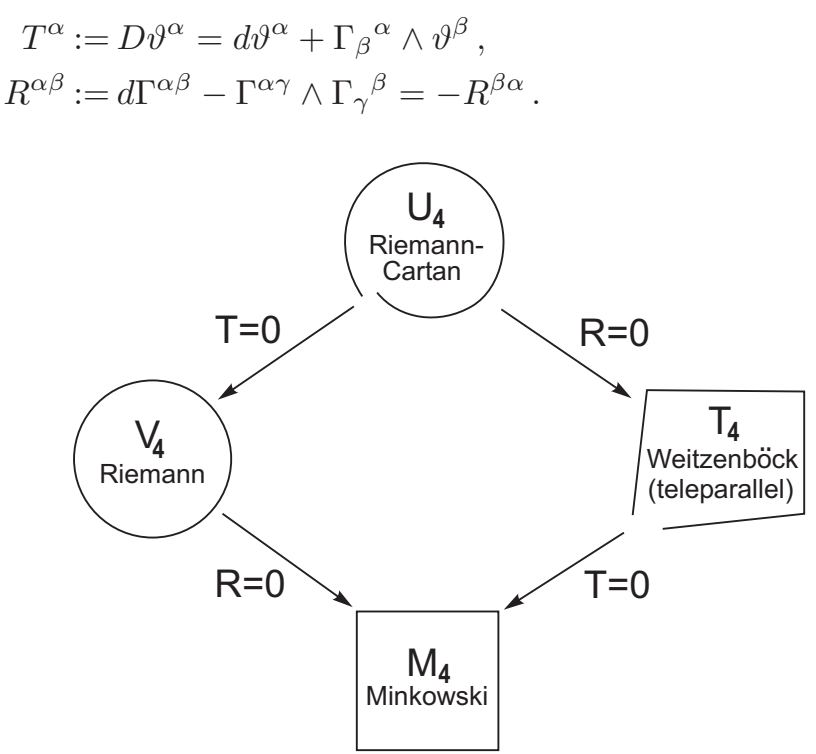

Fig.1. A Riemann-Cartan space $U_{4}$ with torsion $T$ and curvature $R$ and its different limits (nonmetricity vanishes: $\left.Q_{\alpha \beta}:=-D g_{\alpha \beta}=0\right)$, see [5], p.174.

Note that in the term $\Gamma_{\beta}{ }^{\alpha} \wedge \vartheta^{\beta}$ of (3) the rotations and translation mix algebraically, due to the semi-direct product structure. Hence it has to be taken with a grain of salt that $T^{\alpha}$ is called the translation field strength. In (4), the second term on the right-hand-side $-\Gamma^{\alpha \gamma} \wedge \Gamma_{\gamma}{ }^{\beta}$ is due to the 
non-commutative structure of the Lorentz rotations: they form a non-Abelian sub-algebra of the Poincaré algebra.

The different limits of a RC space are represented in Fig.1. GR takes place in a $V_{4}, \mathrm{PG}$ in a $U_{4}, \mathrm{GR}_{\|}$in a $T_{4}$, and, when gravity can be neglected, we are in an $M_{4}$.

The definition (3) of the torsion, written with respect to coordinates, degenerates to (1). Moreover, the explicit form of the Lorentz connection, spelled out in coordinate indices, is $\Gamma_{i j}{ }^{k}=\widetilde{\Gamma}_{i j}{ }^{k}-K_{i j}{ }^{k}$, with the contortion tensor

$$
K_{i j}{ }^{k}=-\frac{1}{2}\left(T_{i j}{ }^{k}-T_{j}{ }^{k}{ }_{i}+T^{k}{ }_{i j}\right)=-K_{i}{ }^{k}{ }_{j} .
$$

So much about the geometry of the PG.

The physics of the PG is determined by a Lagrange 4form

$$
L=V\left(g_{\alpha \beta}, \vartheta^{\alpha}, T^{\alpha}, R^{\alpha \beta}\right)+L_{\text {mat }}\left(g_{\alpha \beta}, \vartheta^{\alpha}, \Psi, D \Psi\right) .
$$

$V$ is the gravitational gauge part of the Lagrangian, depending on the geometrical field variables, $L_{\text {mat }}$ is the matter Lagrangian depending on some minimally coupled matter fields $\Psi(x)$, a Dirac field, for example. For special considerations referring to nonminimal coupling, compare Sec. 8.

By varying with respect to the gauge potentials ( $\delta$ denotes a variation), we can read off the sources in the field equations of the $\mathrm{PG}$ as

$\mathfrak{T}_{\alpha}=\frac{\delta L_{\mathrm{mat}}}{\delta \vartheta^{\alpha}} \quad$ and $\quad \mathfrak{S}_{\alpha \beta}=\frac{\delta L_{\mathrm{mat}}}{\delta \Gamma^{\alpha \beta}}=-\mathfrak{S}_{\beta \alpha}$,

respectively. They turn out to be the canonical 3 -forms of energy-momentum $\mathfrak{T}_{\alpha}$ and of spin angular momentum $\mathfrak{S}_{\alpha \beta}$ of matter. ${ }^{1}$

We postpone the discussion of the explicit form of the gravitational Lagrangian $V$ since this is not necessary for the understanding of the equations of motion of test particles in PG. We will only use it later in order to see that PG embodies viable gravitational theories, namely GR, Einstein-Cartan theory, and the teleparallel equivalent of GR.

\section{How does one measure torsion of spacetime?}

We have now a general idea how a PG looks like. We recognize that $P G$ is a straightforward extension of $G R$, and we wonder, how a test particle moves in a spacetime with torsion.

Clearly, we will take recourse to the established methods of GR. GR is the only theory of nature in which the motion of a test particle is a consequence of the field equation of that theory and of the energy-momentum law following therefrom. Thus, $D \mathfrak{t}_{\alpha}=0$, the energy-momentum law of matter in GR, yields the motion of the momentum vector of a test particle, see, for instance, the textbook of Papapetrou

\footnotetext{
1 They translate into the corresponding quantities of tensor analysis as follows: $\mathfrak{T}_{\alpha}=\mathcal{T}_{\alpha}{ }^{\beta} \epsilon_{\beta}$ and $\mathfrak{S}_{\alpha \beta}=\mathcal{S}_{\alpha \beta}{ }^{\gamma} \epsilon_{\gamma}$, with the 3-form density $\left.\epsilon_{a}:=e_{\alpha}\right\rfloor \epsilon$, the frame $e_{\alpha}$, and the volume 4-form density $\epsilon$. In the reverse order, we have $\vartheta^{\beta} \wedge \mathfrak{T}_{\alpha}=\epsilon \mathcal{T}_{\alpha}{ }^{\beta}$ and $\vartheta^{\gamma} \wedge \mathfrak{S}_{\alpha \beta}=\epsilon \mathcal{S}_{\alpha \beta}{ }^{\gamma}$.
}

[8], Chapter X (Equations of motion in general relativity). The angular momentum law is trivial in GR. It just entails the symmetry of the energy-momentum tensor of matter.

In PG, there emerges a second field equation of gravity and, induced by it, an independent angular momentum law. Thus, in PG we have the momentum and the angular momentum laws. This coupled set of equations is used as a basis to derive equations of motion for test bodies. Why should there be a reason to change horses that carried us so far in the past? To postulate the equations of motion for a test particle independently of the field equations controlling gravity is potentially dangerous, because it will likely lead to inconsistencies, and it defies established practice in GR.

In other words, the study of the equations of motion of test particles in PG follows the same pattern as in GR. The only differences, see [5], Chapter 14 (Equations of motion), are as follows: The energy-momentum law picks up Lorentz type of forces,

$$
\partial_{j} \mathcal{T}^{i j} \cong\left(K_{j k}{ }^{i}-\widetilde{\Gamma}_{j k}{ }^{i}\right) \mathcal{T}^{j k}-\mathcal{S}^{j k l} R_{j k}{ }^{i} l,
$$

and the angular momentum law, for nonvanishing spin, becomes nontrivial,

$$
\partial_{k} \mathcal{S}^{i j k} \cong \mathcal{T}^{[i j]}+2 \Gamma^{[i}{ }_{k l} \mathcal{S}^{j] k l} .
$$

These equations are here displayed in coordinate language for better comparison with the older literature. They are "weak identities", hence the $\cong$ sign, since we assumed the validity of the field equation for matter: $\delta L_{\text {mat }} / \delta \Psi=0$. We stress that the two theorems (8) and (9) are generally valid in PG independently of the explicit choice of the gravitational Lagrangian $V$ in (6). That is, they apply to all torsion theories that are formulated in a general-relativistic framework.

Let us now list chronologically a selection of decisive papers on the measurement of torsion in order to provide an appropriate background for the evaluation of the papers of Mao et al. [1] and March et al. [4]. In

- 1971: Ponomariev [9] assumed that test particles move along autoparallels (the straightest lines) of the RCspacetime:

$$
\frac{d^{2} x^{k}}{d \tau^{2}}+\Gamma_{i j}{ }^{k} \frac{d x^{i}}{d \tau} \frac{d x^{j}}{d \tau}=0 .
$$

There was no reason given. Of course, from a purely geometrical point of view, these curves have a preferential role in a RC-space. This does not imply, however, that they have to have a preferred role in physics, too. We will see that this assumption reappears in the literature later on. Then, in

- 1971, one of us [10] pointed out that "Torsion can be measured by means of a test particle with spin possessing a canonical energy-momentum tensor with a nonvanishing antisymmetric part." This was derived from the energymomentum law (8) of PG; note that the torsion enters (8) via the contortion $K$, see (5). As far as we know, Ponomariev did not object to this conclusion. In 
- 1975, Adamowicz \& Trautman [11] took the angular momentum law (9) and deduced the spin precession induced by torsion: "The torsion of space-time may be measured by observing the precession of [the] spin of a particle." These two papers set the stage for the application of some more subtle methods. In

-1979, Rumpf [12], in his Erice lecture (given during the beginning of May 1979), computed by a quantum-mechanical method the characteristic precession frequency of a Dirac spin in a torsion field. This gave confidence that the spin precession in a torsion field is a realistic effect, obeyed by one of the fundamental fermionic fields of nature, see also [13]. In

- 1979/80, Stoeger \& Yasskin [14,15] asked the question: "Can a macroscopic gyroscope feel a torsion?". They used the general theory of spin motion of Mathisson \& Papapetrou. Their verdict is unequivocal: "Our results show that the torsion couples to spin but not to rotation. Thus a rotating test body with no net spin will ignore the torsion and move according to the usual Papapetrou equations. Hence the standard tests of gravity are insensitive to a torsion field " (emphasis by us). Is there anything more to be said? This should have been the (definite) end of the story. In

- 1981, Audretsch [16] considered the Dirac electron in a spacetime with torsion. Since Rumpf [12] had used a somewhat unconventional quantum-mechanical procedure, it was reassuring that Audretsch, by employing a WKB approximation of the Dirac equation in lowest order, found the same precession frequency of the spin as Rumpf and the same effective connection for the transport of the spin vector.

The basic understanding of the spin-torsion coupling was clarified at this time (1981). For more details and further literature, we refer to [5], Chapter 14. Still, let us have a quick look at some subsequent papers for curiosity. In

- 1997, Lämmerzahl [17] revisited the Hughes-Drever experiment, which was originally used to exclude a possible anisotropy of the mass. He determined the influence of torsion on the energy levels of the atoms involved and found as upper bound for the axial piece of the torsion ${ }^{(3)} T^{\alpha}<$ $10^{-15}$ meter $^{-1}$.

This result reminds us that the remaining irreducible pieces of the torsion, ${ }^{2}$ namely the tensor piece ${ }^{(1)} T^{\alpha}$ and the vector piece ${ }^{(2)} T^{\alpha}$, with $T^{\alpha}={ }^{(1)} T^{\alpha}+{ }^{(2)} T^{\alpha}+{ }^{(3)} T^{\alpha}$, must be measured by means of test particles with spins $s \neq$ $\frac{1}{2}$. We extract from the results of Seitz [18] and of Spinosa $[19,20]$ the following formula for the torsion as seen by a test spin $\mathrm{s}$, which is valid for $s=\frac{1}{2}, 1, \frac{3}{2}, 2$ :

\footnotetext{
${ }^{2}$ Explicitly, we have for the (co)vector and the axial (co)vector pieces $\left.\mathcal{V}:=e_{\beta}\right\rfloor T^{\beta}$ and $\mathcal{A}:={ }^{\star}\left(\vartheta_{\alpha} \wedge T^{\alpha}\right)$, with ${ }^{(2)} T^{\alpha}=-\frac{1}{3} \mathcal{V} \wedge \vartheta^{\alpha}$ and ${ }^{(3)} T^{\alpha}=\frac{1}{3}{ }^{\star}\left(\mathcal{A} \wedge \vartheta^{\alpha}\right)$, respectively, see [5], p.225.
}

$$
\begin{aligned}
T_{s>0}^{\alpha} & =\left(1-\frac{1}{2 s}\right) T^{\alpha}+\frac{3}{2 s}{ }^{(3)} T^{\alpha} \\
& =\left(1-\frac{1}{2 s}\right)\left({ }^{(1)} T^{\alpha}+{ }^{(2)} T^{\alpha}\right)+\left(1+\frac{1}{s}\right){ }^{(3)} T^{\alpha} .
\end{aligned}
$$

Accordingly, a Proca field, which carries spin 1, couples, in contrast to the Dirac field, to all three pieces of the torsion. Subsequently, in 2008, Kostelecky, Russell, and Tasson [21], by using new data, confirmed the upper bound for a possible torsion. In

- 2000 Kleinert [22], quite surprisingly, "proved", neglecting almost all of the previous literature on equations of motion, that a spinless particle follows an autoparallel path thereby sensing torsion. This contradicts established theories, see our discussion above of Ponomariev (1971) and the consequences.

Kleinert takes an argument from particle physics. The spin 1 of a rho vector-meson, if considered as a bound state on a quark level, may be only caused by orbital angular momentum, that is, the spin 1 of the $\rho(770)$ may be orbital angular momentum in camouflage. However, this argument forgets the lesson of effective field theory. ${ }^{3}$

If a $\rho(770)$ moves at moderate speed in an exterior gravitational field, there is every reason to believe that it does behave like a Proca field of spin 1, in accordance with its classification in elementary particle tables. In fact, we know experimentally in the case of a neutron, moving in a gravitational field, that it behaves like a Dirac particle of spin $\frac{1}{2}$, see the Colella-Overhauser-Werner (COW) experiment [23] and its interpretation [24,25]. Whether the neutron spin $\frac{1}{2}$ has, on the quark level, an orbital angular momentum contribution of the three quarks, has no relevance for the COW-type experiment. If we are in a quark-gluon plasma, however, then the quark spin is of relevance and torsion couples to it, but under normal conditions the neutron is of $\operatorname{spin} \frac{1}{2}$.

Accordingly, Kleinert's new universality principle of orbital and spin angular momentum mixes up different levels of observation. Above all, Kleinert's conclusion on the autoparallels drawn therefrom, as we saw above, defies all knowledge on equations of motion in general-relativistic field theories. His nuclear physics arguments are contrived and do not apply to the neutrons of the COW experiment nor to the $\rho(770)$. In

- 2002 Shapiro [26], in his extended review of torsion, found again equations of motion for a spin in a torsion field that are consistent with those of Rumpf, Audretsch, and Lämmerzahl mentioned above, see [26], Eq. (4.60). However, in

\footnotetext{
3 "In physics, an effective field theory is, as any effective theory, an approximate theory, (usually a quantum field theory) that includes appropriate degrees of freedom to describe physical phenomena occurring at a chosen length scale, while ignoring substructure and degrees of freedom at shorter distances (or, equivalently, at higher energies)", see Wikipedia of 30 March 2013.
} 
- 2007, Mao, Tegmark, Guth, Cabi ${ }^{4}$ [1], in their investigation on the possible effect of torsion on the rotating quartz balls of the gyroscope of the Gravity Probe B experiment [2], proposed two postulates: (i) The equation of motion for their "spin", see [1], Eq. (19), and (ii) their spin has to move along an autoparallel. Both postulates are ad hoc and the second one is even inconsistent, as we saw above. We will come back to their paper in Sec. 9. Already before the final publication of the Mao et al. paper, in

- 2007, Flanagan \& Rosenthal [27] noted that the gravitational theory with torsion, taken by Mao et al. as a guinea pig, is inconsistent. This led Mao et al. to declare that the torsion theory they used should "...not be viewed as a viable physical model, but as a pedagogical toy model giving concrete illustrations of the various effects and constraints that we discuss." Why should an inconsistent model be good enough for pedagogical purposes if it is basically not good enough for a scientific journal?

At the same time Flanagan \& Rosenthal [27] stated in their conclusions that "There may exist other torsion theories which could be usefully constrained by GPB. It would be interesting to find such theories." In Sec. 9 we will show that such a finding appears to be only a very remote possibility. Shortly afterwards, in

- 2007/08, Puetzfeld \& Obukhov [28,29] showed by a multipolar approximation scheme that Mao et al. are ruled out for a very large class of theories, only intrinsic spin couples to torsion, in particular it was explicitly shown, see Sec. 10 of [29], that the model of Hayashi and Shirafuji [30] does not have the properties claimed by Mao et al.. In

- 2010, Babourova \& Frolov [31] came up with an alternative gravitational theory in which they claim that orbital angular momentum can be a source of torsion. However, their construct is inconsistent since already their Lagrangian [31], Eq. (10), depends on the position "vector" and is as such no longer a covariant quantity. Still, in

- 2011, March et al. [4] reiterate Mao et al., but take instead Mercury and Moon data and, later, data of the Lageos satellite. Again they "...make use of the autoparallel trajectories, which in general differ from geodesics when torsion is present." We saw already in the context of the Mao et al. discussion that this leads to nowhere.

\section{Poincaré gauge theory, its general structure, quadratic gauge Lagrangians}

Let us now come back to the PG, which we only sketched in Sec. 2. Consider the minimally coupled total Lagrangian

\footnotetext{
4 In 2006, when the Mao et al. paper was uploaded to arXiv.org, one of us immediately communicated his objections to Max Tegmark and his coauthors, basically the same objections as those to be discussed in this Letter; but it was of no avail. The analogous happened in the case of the March et al. papers. They did not find our arguments convincing either.
}

$L$ in (6). Since we want to leave the gravitational Lagrangian $V=V\left(g_{\alpha \beta}, \vartheta^{\alpha}, T^{\alpha}, R^{\alpha \beta}\right)$ open for the time being, we define the translational and Lorentz field excitations (or field momenta),

$H_{\alpha}=-\frac{\partial V}{\partial T^{\alpha}}, \quad H_{\alpha \beta}=-\frac{\partial V}{\partial R^{\alpha \beta}}$.

As soon as we specify the explicit form of $V$, we can compute the H's simply by partial differentiation.

The field equations of the PG read

$$
\begin{aligned}
D H_{\alpha}-t_{\alpha} & =\mathfrak{T}_{\alpha} & & \left(\text { First grav. FEQ }, \delta / \delta \vartheta^{\alpha}\right), \\
D H_{\alpha \beta}-s_{\alpha \beta} & =\mathfrak{S}_{\alpha \beta} & & \left(\text { Second grav. FEQ }, \delta / \delta \Gamma^{\alpha \beta}\right), \\
\delta L_{\text {mat }} / \delta \Psi & =0 & & \text { (Matter FEQ } \delta / \delta \Psi)
\end{aligned}
$$

for their derivation see [5], Chapter 5, and the references given there. The sources on the right-hand-side of First and Second are the canonical energy-momentum $\mathfrak{T}_{\alpha}$ and spin $\mathfrak{S}_{\alpha \beta}$ of matter defined in Eq. (7). The energy-momentum and spin of the gauge fields are, respectively,

$$
\begin{aligned}
t_{\alpha} & \left.\left.\left.:=e_{\alpha}\right\rfloor V+\left(e_{\alpha}\right\rfloor T^{\beta}\right) \wedge H_{\beta}+\left(e_{\alpha}\right\rfloor R^{\beta \gamma}\right) \wedge H_{\beta \gamma}, \\
s_{\alpha \beta} & :=-\vartheta_{[\alpha} \wedge H_{\beta]} .
\end{aligned}
$$

As we shall see later in detail, we will find the Einstein sector of the PG if $H_{\alpha}=0$ and thus First degenerates to the innocently looking $-t_{\alpha}=\mathfrak{T}_{\alpha}$.

Like in electrodynamics and in Yang-Mills theory, the gauge field Lagrangian should be algebraic in the field strengths, here in $T^{\alpha}$ and $R^{\alpha \beta}$. Then we find second order partial differential equations (PDEs) in the gauge variables $\left(\vartheta^{\alpha}, \Gamma^{\alpha \beta}\right)$. Moreover, it should be quadratic in order to induce quasi-linearity of the PDEs and thus wave type equations for both gravitational field equations.

Symbolically, such a quadratic gauge Lagrangian, with the conventional "weak" gravitational constant $\kappa$, the "strong" gravitational constant $\varrho$, and the cosmological constant $\Lambda_{0}$, reads

$$
V_{\mathrm{qPG}} \sim \frac{1}{\kappa}\left(R+X+\Lambda_{0}+\{T\}^{2}\right)+\frac{1}{\varrho}\{R\}^{2} .
$$

Here $R$ denotes the curvature scalar and $X \sim \epsilon^{i j k l} R_{[i j k l]}$ the curvature pseudoscalar, $\{T\}^{2}$ symbolizes the sum of four torsion square pieces and $\{R\}^{2}$ the sum of eight curvature square pieces. The exact formula, which we do not need here, can be found in Baekler et al. [32] or in [5], Eq. (5.13).

\section{Poincaré gauge theory and its viable subsets}

The quadratic gauge Lagrangian (18), taken together with the field equations (13), (14), and (15), represent the output of gauging the Poincaré group according to the conventional gauge principles. This we consider to be the class of standard torsion theories. To select a definite gauge Lagrangian $V_{\mathrm{qPG}}$ will be a task for the future, for details see [5], Part B. 
The simplest Lagrangian is the curvature scalar of the RC-spacetime yielding the Einstein-Cartan theory of gravity (EC), see Figure 2:

$$
\begin{aligned}
V_{\mathrm{EC}} & \sim \frac{1}{\kappa} R \sim \frac{1}{\kappa} \star\left(\vartheta^{\alpha} \wedge \vartheta^{\beta}\right) \wedge R_{\alpha \beta}(\Gamma) \\
& \sim \frac{1}{\kappa} e_{i}{ }^{\alpha} e_{j}{ }^{\beta} R^{i j}{ }_{\alpha \beta}(\Gamma) \quad\left({ }^{\star}=\text { Hodge dual }\right) .
\end{aligned}
$$

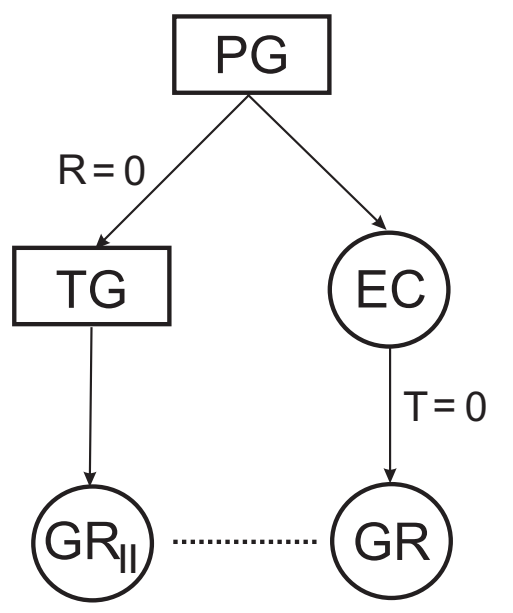

Fig,2. Classification of Poincaré gauge theories of gravity (Blagojević, Hehl, Obukhov, see the frontispiece of [5]): $\mathbf{P G}=$ Poincaré gauge theory (of gravity), $\mathbf{E C}=$ EinsteinCartan(-Sciama-Kibble) theory (of gravity), GR = general relativity (Einstein's theory of gravity), $\mathbf{T G}=$ translation gauge theory (of gravity) aka teleparallel theory (of gravity), $\mathbf{G R}_{\|}=$a specific TG known as teleparallel equivalent of GR (spoken "GR teleparallel"). The symbols in the figure have the following meaning: rectangle $\square \rightarrow$ class of theories; circle $\bigcirc \rightarrow$ definite viable theories.

The EC can be put in such a form that it is represented by GR plus a weak gravitational spin-spin contact interaction that only leads to deviations from GR at extremely high matter densities. The critical density at which GR breaks down is

$\rho_{\text {crit }} \sim m /\left(\lambda_{\text {Compton }} \ell_{\text {Planck }}^{2}\right)$.

For a nucleon this is more than $10^{52} \mathrm{~g} / \mathrm{cm}^{3}$ or $10^{24} \mathrm{~K}$, with a critical length of $\ell_{\text {crit }} \sim 10^{-26} \mathrm{~cm}$. Thus, EC is a viable gravitational theory. In cosmological applications it could be of relevance. If in EC the material spin $\mathfrak{S}$ and thus the torsion vanish, we recover GR, see Figure 2. Consequently, PG contains the viable theories EC and GR and is as such a non-empty framework with a reasonable GR limit.

Perhaps surprisingly, PG has a further subclass of a viable gravitational theory. If we choose in the Lagrangian (18) the option with $\sim \frac{1}{\kappa}\{T\}^{2}$ and require the vanishing of the curvature, that is, we imbed this Lagrangian in a Weitzenböck spacetime, then we have the Lagrangian of a translational gauge theory of gravity (TG), namely

$V_{\mathrm{TG}} \sim \frac{1}{\kappa}\{T\}^{2}+R_{\alpha}^{\beta} \wedge \lambda_{\beta}^{\alpha}$ here $\lambda^{\alpha}{ }_{\beta}$ is a Lagrange multiplier, see [5], Chapter 6, for details and literature.

Now we require additionally local Lorentz invariance and can single out a definite version of $\{T\}^{2}$, namely

$$
\begin{aligned}
V_{\mathrm{GR}_{\|}=} & -\frac{1}{2 \kappa} T^{\alpha} \wedge^{\star}(-\underbrace{{ }^{(1)} T_{\alpha}}_{\text {tensor }}+2 \underbrace{{ }^{(2)} T_{\alpha}}_{\text {vector }}+\frac{1}{2} \underbrace{{ }^{(3)} T_{\alpha}}_{\text {axial vec. }}) \\
& +R_{\alpha}{ }^{\beta} \wedge \lambda^{\alpha}{ }_{\beta},
\end{aligned}
$$

the teleparallel equivalent of GR. For scalar and for Maxwell matter, that is, for $\mathfrak{T}_{i j}=\mathfrak{t}_{i j}$, it can be shown that $\mathrm{GR}_{\|}$and GR are equivalent, see [5], Chapter 6, and as well the recent reviews of Aldrovandi \& Pereira [33] and Maluf [34]. Hence we found another viable version of PG, demonstrating the power of this framework.

\section{The universally valid energy-momentum law in Poincaré gauge theory}

We generalize the corresponding law of $\mathrm{GR}, D \mathfrak{t}_{\alpha}=0$, together with the symmetry condition $\vartheta_{[\alpha} \wedge \mathfrak{t}_{\beta]}=0$, to the energy-momentum law and angular momentum law of PG:

$$
\begin{aligned}
D \mathfrak{T}_{\alpha} & \left.\left.\cong\left(e_{\alpha}\right\rfloor T^{\beta}\right) \wedge \mathfrak{T}_{\beta}+\left(e_{\alpha}\right\rfloor R^{\beta \gamma}\right) \wedge \mathfrak{S}_{\beta \gamma}, \\
D \mathfrak{S}_{\alpha \beta} & \cong-\vartheta_{[\alpha} \wedge \mathfrak{T}_{\beta]} .
\end{aligned}
$$

These are the laws in exterior form calculus, for the tensor analysis version, see (8) and (9). Incidentally, Cartan assumed ad hoc that the right-hand-side of (23) has to vanish, similar as in GR's law $D \mathfrak{t}_{\alpha}=0$. However, if one does the Noether "algebra" correctly, one finds (23), indeed; see, for instance, [35], Eq. (5.2.10) for $Q_{\alpha \beta}=0$, or [36], Eq. (4.11). Interestingly enough, in 3 dimensions, Cartan's assumption turns out to be correct.

In order to isolate the torsion-dependent terms, we decompose the connection $\Gamma^{\alpha \beta}=\widetilde{\Gamma}^{\alpha \beta}-K^{\alpha \beta}$ into its Riemannian part $\widetilde{\Gamma}^{\alpha \beta}$ plus torsion-dependent pieces. The contortion $K^{\alpha \beta}$ is defined in (5); furthermore, we have $T^{\alpha}=$ $K^{\alpha}{ }_{\beta} \wedge \vartheta^{\beta}$. The split connection will be substituted into the $D$ and $R^{\beta \gamma}$ of (23). We find ( $\mathcal{L}=$ Lie derivative),

$$
\begin{gathered}
\left.\widetilde{D}\left[\mathfrak{T}_{\alpha}-\mathfrak{S}^{\beta \gamma}\left(e_{\alpha}\right\rfloor K_{\beta \gamma}\right)-\mathfrak{S}^{\beta \gamma} \wedge\left(\mathcal{L}_{e_{\alpha}} K_{\beta \gamma}\right)\right] \\
\left.\cong \mathfrak{S}^{\beta \gamma} \wedge\left(e_{\alpha}\right\rfloor \widetilde{R}_{\beta \gamma}\right) .
\end{gathered}
$$

This is a universally valid law for all PGs, independent of the gravitational Lagrangian $V$. It shows conclusively that spin alone does couple to contortion and hence to torsion. Note that its right-hand-side depends only on the Riemannian piece, in contrast to Eq. (8), which depends on the complete RC-curvature.

For vanishing torsion, we have $\left.\widetilde{D} \mathfrak{T}_{\alpha}=\mathfrak{S}^{\beta \gamma} \wedge\left(e_{\alpha}\right\rfloor \widetilde{R}_{\beta \gamma}\right)$, that is, a momentum law that exhibits a spin-curvature force density on its right-hand-side. This is the same structure as the Mathisson-Papapetrou force in GR, with the difference that here the force density does not contain integrated moments. Then, $\widetilde{D} \mathfrak{t}_{\alpha}=0$, with $\mathfrak{t}_{\alpha}=\mathfrak{T}_{\alpha}-\widetilde{D} \mu_{\alpha}$ and $\left.\left.\left.\mu_{\alpha}=-2 e_{\beta}\right\rfloor \mathfrak{S}_{\alpha}{ }^{\beta}+\frac{1}{2} \vartheta_{\alpha} \wedge\left(e_{\beta}\right\rfloor e_{\gamma}\right\rfloor \mathfrak{S}^{\beta \gamma}\right)$. 
In PG, integrate (25) over a drop of a spin fluid. Then we find the equation of motion of the momentum of the spin drop. It will certainly not be an autoparallel curve, see [29].

\section{On nonminimal coupling}

In this Letter we have confined our discussion to matter interacting minimally with the gravitational field. However, currently considerable attention is drawn to models with nonminimal gravitational coupling see [37-39], for example. In particular, in some theories such a generalized interaction arises when the gravitational coupling constant is replaced by a coupling function $F$ that depends on the gravitational field strength (curvature and/or torsion). The corresponding modified Lagrangian reads $F L_{\text {mat }}$. One can verify that the metrical energy-momentum tensor $\sqrt{-g} \mathfrak{t}_{i j}=2 \delta\left(\sqrt{-g} L_{\mathrm{mat}}\right) / \delta g^{i j}$ even for the spinless matter is not covariantly conserved $[40,37]$, but instead it satisfies the balance law

$\nabla^{i} \mathfrak{t}_{i j}=-\left(g_{i j} L_{\text {mat }}+\mathfrak{t}_{i j}\right) \frac{\nabla^{i} F}{F}$.

As an immediate consequence, we find that the motion of a test particle or body is non-geodetic since an extra force is acting on it, which is proportional to the gradient of the coupling function $F$.

It was demonstrated in [39] that the modified conservation law (26) leads to non-geodetic motion, in which $F$ can depend arbitrarily on the components of the Riemann curvature tensor (in practice, being a scalar, $F$ is any function of all possible algebraic invariants built from the curvature). Furthermore, in [41] it was shown that the remarkably simple law (26) is also true for the coupling function $F$ that depends arbitrarily on the Riemann-Cartan curvature and torsion tensors.

This implies that a nonminimal coupling of matter to gravity represents actually a loophole that allows to detect the possible non-Riemannian structure of spacetime by means of spinless matter. Such a loophole, however, is qualitatively different from the "Hypothesis T" which we discuss in the next section.

\section{Our answer to Mao et al. and March et al.}

In a discussion, Tegmark made an attempt to elucidate the Mao et al. philosophy by defining the Hypothesis $T$ [42]: "There's a consistent nonstandard gravity theory where torsion couples to macroscopic rotation". Taking this hypothesis as their starting point, Mao et al. believe that they could constrain the possible torsion of spacetime in such a nonstandard gravity theory via the experimental results of GPB.

If we take PG as the standard torsion theory, then rotating quartz balls are blind to torsion, as we underlined again in Sec. 7. Even if we do not commit ourselves to a specific form of the gravitational Lagrangian $V$, the energy- momentum law commands that torsion only couples to spin, see equation (25) and the two terms that couple spin $\mathcal{S}$ directly to the contortion $K$.

Moreover, as Flanagan \& Rosenthal [27] and two of us [29] have shown, the teleparallel theory used by Mao et al. also cannot provide a framework for measuring torsion. Hence Hypothesis T is empty so far. Furthermore, Mao et al. postulated the autoparallel as an equation of motion, which is incorrect in a general-relativistic set-up anyway, quite independent of the field equations.

It is a general rule in physics that one can only measure a certain quantity provided one has a consistent theory about this quantity in the first place. If one wants to measure, say, an acceleration of a particle, one has first to define an acceleration via $\boldsymbol{a}=d^{2} \boldsymbol{x} / d t^{2}$. After these considerations, one can measure $\boldsymbol{a}$. A sensible interpretation of experiments in physics usually requires preceding theoretical groundwork. By the same token, we have first to develop a consistent theory of the torsion before we are able to measure it.

But there is even a more direct argument that is lethal to Hypothesis T: In the whole of the Mao et al. paper, the authors only speak about the vacuum field equations. They never address the question of how a field equation could look like where "macroscopic rotation", that is, macroscopic orbital angular momentum features as a source of a gravitational field equation. Mao et al. claim that in other papers there is an "assumption that orbital angular momentum cannot be the source of torsion." In fact, orbital angular momentum as a tensor is only known for extended structures, never as a density existing at one point. It is a quantity alien to local field theory. The torsion tensor, however, is a local point-dependent object. Accordingly, in a local field equation, these two quantities cannot be related to each other. That is, orbital angular momentum cannot be the source of torsion on account of the different nature of those two objects.

In special relativity in Cartesian coordinates, the orbital angular momentum flux density is $x_{[i} \mathcal{T}_{j]}{ }^{k}$, with the position vector $x_{i}$ and the energy-momentum tensor $\mathcal{T}_{i}{ }^{j}$. The divergence of the orbital angular momentum flux density reads

$\partial_{k}\left(x_{[i} \mathcal{T}_{j]}^{k}\right)=\mathcal{T}_{[j i]}+x_{[i \mid} \partial_{k} \mathcal{T}_{\mid j]}^{k}$.

If the action of a physical system without (intrinsic) spin angular momentum is invariant under spacetime translations and (Lorentz) rotations, the energy-momentum is conserved, $\partial_{k} \mathcal{T}_{i}{ }^{k}=0$, and we find the angular momentum conservation law

$\partial_{k}\left(x_{[i} \mathcal{T}_{j]}^{k}\right)=\mathcal{T}_{[j i]}=0$.

The orbital angular momentum $x_{[i} \mathcal{T}_{j]}{ }^{k}$ is not a tensor in curvilinear coordinates. In the words of Truesdell [43], it is a quantity that is "not indifferent" to coordinate transformations, whereas legitimate field-theoretical quantities should be indifferent. Accordingly, this quantity does not exist as a local quantity in a curved (in particular, in Riemann- 
Cartan) spacetime. However, the conservation law of the angular momentum, namely $\mathcal{T}_{[j i]}=0$, can be generalized to Riemann-Cartan spacetimes, provided the momentum law is fulfilled. When, in addition, matter possesses the intrinsic spin angular momentum $\mathcal{S}_{i j}{ }^{k}$, it contributes to the balance law the divergence $D_{k} \mathcal{S}_{i j}{ }^{k}$ thus providing the total angular momentum law $D_{k} \mathcal{S}_{i j}{ }^{k}-\mathcal{T}_{[i j]}=0$. The latter is meaningful even if the orbital angular momentum does not exist in curved space: In contrast to its orbital partner, spin angular momentum $\mathcal{S}_{i j}{ }^{k}$ is a well-defined "indifferent" tensor in a Riemann-Cartan spacetime and can act as a source.

Hypothesis $\mathrm{T}$ is untenable, since it links the field theoretical notion "torsion" with the orbital angular momentum of an extended structure; this net orbital angular momentum cannot be represented as an integral over a local orbital angular momentum density, since such a density does not exist. We thus conclude that Hypothesis $\mathrm{T}$ is empty.

No counterexample is known to our result. Our conclusion can be found in the last phrase of our Abstract.

Eventually, we have also an optimistic message: Already in 1983, Ni [44] suggested to build gyroscopes with spinpolarized balls as active elements consisting of solid heliumthree $\left({ }^{3} \mathrm{He}\right)$ and to put them into orbit around the Earth. $\mathrm{Ni}$ has also used for experiments in the gravitational field dysprosium-iron compounds $\mathrm{Dy}_{6} \mathrm{Fe}_{23}$, see [45], with a relatively high net spin of about 0.4 electron spins per atom, but with no disturbing magnetic moment. With such tools one could hope to find torsion, if it exists in nature.

\section{Acknowledgments}

One of us (F.W.H.) is grateful to Max Tegmark and also to Yi Mao for an extended and lively email discussion; even though we could not agree on a joint position, this discussion was very helpful. Likewise we would like to thank Giovanni Bellettini and Riccardo March for several discussions. We thank Milutin Blagojević for the drawing of Figure 2. Moreover, we thank Wei-Tou Ni, Neil Russell, Helmut Rumpf, and Francis Everitt for useful remarks. F.W.H. was partially supported by the German-Israeli Foundation for Scientific Research and Development (GIF), Research Grant No. 1078-107.14/2009. D.P. was supported by the Deutsche Forschungsgemeinschaft (DFG) through the grant LA-905/8-1.

\section{References}

[1] Y. Mao, M. Tegmark, A. Guth, and S. Cabi, Constraining torsion with Gravity Probe B, Phys. Rev. D 76, 104029 (2007).

[2] C. W. F. Everitt, D. B. DeBra, B. W. Parkinson, J. P. Turneaure, J. W. Conklin, M. I. Heifetz, G. M. Keiser and A. S. Silbergleit et al., Gravity Probe B: Final results of a space experiment to test general relativity, Phys. Rev. Lett. 106, 221101 (2011) [arXiv: 1105.3456].

[3] Francis Everitt, private communication by email (April 2013).

[4] R. March, G. Bellettini, R. Tauraso, and S. Dell'Agnello, Constraining spacetime torsion with the Moon and Mercury, Phys. Rev. D 83, 104008 (2011); Constraining spacetime torsion with LAGEOS, Gen. Relativ. Gravit. 43, 3099-3126 (2011) [arXiv:1101.2791].

[5] M. Blagojević and F. W. Hehl (eds.), Gauge Theories of Gravitation. A Reader with Commentaries (Imperial College Press, London, 2013).

[6] D. W. Sciama, On the analogy between charge and spin in general relativity, in: Recent Developments in General Relativity, Festschrift for Infeld (Pergamon Press, Oxford; PWN, Warsaw, 1962) pp. 415-439.

[7] T. W. B. Kibble, Lorentz invariance and the gravitational field, J. Math. Phys. 2, 212-221 (1961).

[8] A. Papapetrou, Lectures on General Relativity (Reidel, Dordrecht, Holland, 1974).

[9] V. N. Ponomariev, Observable effects of torsion in space-time, Bull. Acad. Pol. Sci., Sér. Sci. Math. Astron. Phys. 19, 545-550 (1971).

[10] F. W. Hehl, How does one measure torsion of space-time? Phys. Lett. A 36, 225-226 (1971).

[11] W. Adamowicz and A. Trautman, The principle of equivalence for spin, Bull. Acad. Pol. Sci., Sér. Sci. Math. Astron. Phys. 23, 339-342 (1975).

[12] H. Rumpf, Quasiclassical limit of the Dirac equation and the equivalence principle in the Riemann-Cartan geometry, in: Cosmology and Gravitation: Spin, Torsion, Rotation and Supergravity, P. G. Bergmann and V. de Sabbata, eds. (Plenum, New York, 1980) pp. 93-104.

[13] H. Rumpf, Supersymmetric Dirac particles in Riemann-Cartan space-time, Gen. Rel. Grav. 14, no.9, 773-792 (1982).

[14] W. R. Stoeger and P. B. Yasskin, Can a macroscopic gyroscope feel a torsion? Gen. Rel. Grav. 11, 427-431 (1979).

[15] P. B. Yasskin and W. R. Stoeger, Propagation equations for test bodies with spin and rotation in theories of gravity with torsion, Phys. Rev. D 21, 2081-2094 (1980).

[16] J. Audretsch, Dirac electron in space-times with torsion: Spinor propagation, spin precession, and nongeodesic orbits, Phys. Rev. D 24, 1470-1477 (1981); Erratum Phys. Rev. D 25, 605 (1982).

[17] C. Lämmerzahl, Constraints on space-time torsion from HughesDrever experiments, Phys. Lett. A 228, 223-231 (1997) [grqc/9704047].

[18] M. Seitz, Proca field in a spacetime with curvature and torsion, Class. Quantum Grav. 3, 1265-1273 (1986).

[19] R. Spinosa, Proca test field in a space-time with torsion, Class. Quantum Grav. 4, 473-484 (1987).

[20] R. Spinosa, Spin 3/2 test field in a space-time with torsion, Class. Quantum Grav. 4, 1799-1808 (1987).

[21] V. A. Kostelecky, N. Russell and J. Tasson, New constraints on torsion from Lorentz violation, Phys. Rev. Lett. 100, 111102 (2008).

[22] H. Kleinert, Universality principle for orbital angular momentum and spin in gravity with torsion, Gen. Rel. Grav. 32, 1271-1280 (2000) [gr-qc/9807021].

[23] R. Colella, A.W. Overhauser, and S. A. Werner, Observation of gravitationally induced quantum interference, Phys. Rev. Lett. 34, 1472-1474 (1975).

[24] H. Rauch and S. A. Werner, Neutron Interferometry: Lessons in Experimental Quantum Mechanics (Oxford University Press, Oxford, 2000).

[25] Yu. A. Alexandrov, Fundamental Properties of the Neutron, translated by T. F. Drozdova (Clarendon Press, Oxford, 1992).

[26] I. L. Shapiro, Physical aspects of the space-time torsion, Phys. Rept. 357, 113-213 (2002).

[27] E. E. Flanagan and E. Rosenthal, Can gravity probe B usefully constrain torsion gravity theories? Phys. Rev. D 75, 124016 (2007) [arXiv: 0704.1447].

[28] D. Puetzfeld and Yu. N. Obukhov, Propagation equations for deformable test bodies with microstructure in extended theories of gravity, Phys. Rev. D 76, 084025 (2007); Erratum: Phys. Rev. D 79, 069902 (2009). 
[29] D. Puetzfeld and Yu. N. Obukhov, Probing non-Riemannian spacetime geometry, Phys. Lett. A 372, 6711-6716 (2008).

[30] K. Hayashi and T. Shirafuji, New general relativity, Phys. Rev. D 19, 3524-3553 (1979).

[31] O. V. Babourova and B. N. Frolov, Interaction of the 4-rotational gauge field with orbital momentum, gravidiamagnetic effect, and orbit experiment "Gravity Probe B", Phys. Rev. D 82, 027503 (2010) [arXiv: 1004.1790].

[32] P. Baekler and F. W. Hehl, Beyond Einstein-Cartan gravity: Quadratic torsion and curvature invariants with even and odd parity including all boundary terms, Class. Quantum Grav. 28, 215017 (2011) [arXiv:1105.3504].

[33] R. Aldrovandi and J. G. Pereira, Teleparallel Gravity, An Introduction (Springer, Dordrecht, 2013).

[34] J. W. Maluf, The teleparallel equivalent of general relativity, Annalen der Physik (Berlin) 525, 339-357 (2013) [arXiv:1303.3897].

[35] F. W. Hehl, J. D. McCrea, E. W. Mielke, and Y. Ne'eman, Metric-affine gauge theory of gravity: Field equations, Noether identities, world spinors, and breaking of dilation invariance, Phys. Rept. 258, 1-171 (1995).

[36] Yu. N. Obukhov, Poincaré gauge gravity: Selected topics, Int. J. Geom. Meth. Mod. Phys. 3, 95-138 (2006) [gr-qc/0601090].

[37] O. Bertolami, C. G. Böhmer, T. Harko, and F. S. N. Lobo, Extra force in $f(R)$ modified theories of gravity, Phys. Rev. D 75, 104016 (2007).

[38] S. Nojiri and S. D. Odintsov, Unified cosmic history in modified gravity: from $F(R)$ theory to Lorentz non-invariant models, Phys. Rep. 505, 59-144 (2011).

[39] D. Puetzfeld and Yu. N. Obukhov, Covariant equations of motion for test bodies in gravitational theories with general nonminimal coupling, Phys. Rev. D 87, 044045 (2013).

[40] T. Koivisto, A note on covariant conservation of energymomentum in modified gravities, Class. Quantum Grav. 23, 4289-4296 (2006).

[41] Yu. N. Obukhov and D. Puetzfeld, Conservation laws in gravitational theories with general nonminimal coupling, Phys. Rev. D 87, 081502 (2013) [arXiv:1303.6050].

[42] Max Tegmark, private communication by email (Feb. 2013).

[43] C. Truesdell, Whence the law of moment of momentum? In: Essays in the History of Mechanics by C. Truedell (Springer, New York, 1968) pp. 239-271. We used the German version of this article; therein moment stress and spin angular momentum are additionally discussed: C. Truesdell, Die Entwicklung des Drallsatzes, ZAMM 44, 149-158 (1964).

[44] W.-T. Ni, Spin, torsion and polarized test-body experiments, in: Proc. 1983 Internat. School and Symposium on Precision Measurement and Gravity Experiment, Taipei, ROC, W.-T. Ni, ed. (Nat. Tsing Hua Univ., Hsinchu, Taiwan, ROC, 1983) pp. 532-540; see http://astrod.wikispaces.com/PMGE .

[45] W.-T. Ni, Searches for the role of spin and polarization in gravity, Rep. Prog. Phys. 73, 056901 (2010). 